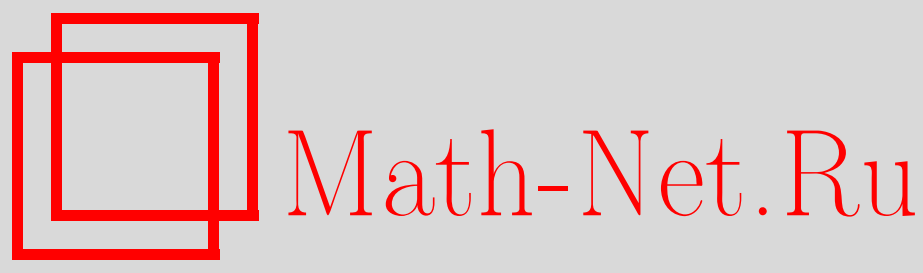

С. В. Смышляев, Об 1-устойчивых совершенно уравновешенных булевых функциях, Дискрет. матем., 2016, том 28, выпуск 2, 117-126

DOI: https://doi.org/10.4213/dm1374

Использование Общероссийского математического портала Math-Net.Ru подразумевает, что вы прочитали и согласны с пользовательским соглашением http: //www . mathnet.ru/rus/agreement

Параметры загрузки:

IP: 18.208 .226 .222

26 апреля 2023 г., $17: 18: 47$

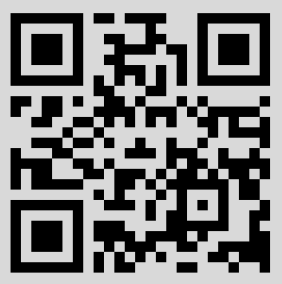




\title{
Об 1-устойчивых совершенно уравновешенных булевых функциях
}

\author{
() 2016 г. $\quad$ С. В. Смышляев*
}

Рассматривается связь между корреляционной иммунностью (устойчивостью) и совершенной уравновешенностью булевых функций. Показано, что для произвольной совершенно уравновешенной булевой функции не выполняется даже некоторое более слабое, чем 1-устойчивость, свойство, что опровергает некоторые результаты Маркуса Дихтла. С другой стороны, доказываются новые утверждения о барьерах совершенно уравновешенных булевых функций, с помощью которых удается доказать, что все совершенно уравновешенные функции, сумма длин барьеров которых меньше числа переменных, являются 1-устойчивыми.

Работа поддержана Российским фондом фундаментальных исследований (проекты 16-01-00226 А и 16-01-00470 А).

Ключевые слова: совершенно уравновешенные функции, барьеры булевых функций, корреляционная иммунность, криптография.

\section{1. Введение}

Важным элементом большого класса криптографических подсистем является набор процедур получения последовательностей, для которых выдвигаются те или иные предположения о случайности. Данные процедуры основываются на использовании доверенных источников случайности (аппаратных датчиков случайных чисел), детерминированных генераторов псевдослучайных чисел и дополнительных случайных данных, возникающих при функционировании системы (например, времен задержек при чтении секторов жесткого диска). При этом при использовании криптосредств в системах, в которых определенная потеря стойкости криптографических механизмов существенно менее критична, чем остановка всей системы, возникает задача обеспечения положительных статистических качеств последовательностей даже в случае сбоев в работе доверенного источника случайности: в случае частичной поломки оборудования требуется не останавливать систему, эксплуатирующую криптосредство, а обеспечивать ее функционирование с сохранением возможного остаточного уровня безопасности.

*Место работы: ООО „КРИПТО-ПРО“, е-mail: smyshsv@gmail.com 
Одним из способов работы с источниками случайности в таких криптографических средствах является следующий: данные, получаемые с доверенного источника случайности, замешиваются с выходами детерминированного генератора псевдослучайных чисел (основанного, например, на регистре сдвига с линейной обратной связью) и дополнительных источников энтропии, после чего обрабатываются фильтрующими примитивами (например, кодирующими устройствами, построенными на основе регистров сдвига и булевых функций). При этом необходимо обеспечивать выполнение следующих двух требований: во-первых, при корректной работе доверенного источника случайности с получением на нем последовательности независимых равновероятных двоичных случайных величин (в зарубежных публикациях употребляется жаргонное выражение „рure random sequence“ - „истинно случайная последовательность“) выходная последовательность должна иметь наилучшие статистические свойства; а во-вторых, в случае сбоя и вырождения последовательности от доверенного источника случайности необходимо по возможности скрыть структурные связи выходов детерминированного генератора псевдослучайных чисел и дополнительных источников случайности. В случае использования кодирующих устройств на основе регистров сдвига и булевых функций первое из данных требований означает совершенную уравновешенность используемых функций, а второе накладывает ограничения на такие параметры функции, как алгебраическая степень, нелинейность, уровень аффинности, порядок корреляционной и алгебраической иммунности (см. [6]). Важной задачей является синтез совершенно уравновешенных функций, обладающих также и другими положительными криптографическими качествами.

Одним из направлений исследований в данной тематике является анализ связи свойства совершенной уравновешенности (для которого, в частности, необходима уравновешенность) с другими свойствами, тоже являющимися более сильными, чем уравновешенность самой функции - такими, например, как уравновешенность набора ее подфункций. К сожалению, на данном пути зарубежными исследователями получено несколько неверных результатов, что, в частности, обсуждалось в [4]. В настоящей работе доказаны утверждения об уравновешенности подфункций совершенно уравновешенных булевых функций. Одно из них показывает существование широкого подмножества совершенно уравновешенных булевых функций, для каждой из которых сразу несколько её подфункций, полученных фиксацией одной из переменных нулем, не являются совершенно уравновешенными - вопреки теории, изложенной в работе Маркуса Дихтла [2]. Другой основной результат, полученный в данной работе, является первым на пути изучения связи между свойствами совершенно уравновешенных булевых функций и корреляционной иммунностью (устойчивостью) функций: доказано, что все совершенно уравновешенные булевы функции, сумма длин барьеров которых меньше числа переменных, являются 1-устойчивыми.

\section{2. Обозначения и определения}

Для любого натурального $n$ множество двоичных наборов длины $n$ будем обозначать через $V_{n}=\{0,1\}^{n}$; множество булевых функций от $n$ переменных - через $\mathcal{F}_{n}$. 
Пусть $n \geqslant 1, l \geqslant 1, f \in \mathcal{F}_{n}$. Рассмотрим систему булевых уравнений

$$
f\left(x_{s}, x_{s+1}, \ldots, x_{s+n-1}\right)=y_{s}, s=1,2, \ldots, l .
$$

Через $f_{l}$ будем обозначать отображение из $V_{l+n-1}$ в $V_{l}$ следующего вида:

$$
f_{l}\left(x_{1}, x_{2}, \ldots, x_{l+n-1}\right)=\left(f\left(x_{1}, \ldots, x_{n}\right), f\left(x_{2}, \ldots, x_{n+1}\right), \ldots, f\left(x_{l}, \ldots, x_{l+n-1}\right)\right) .
$$

Легко видеть, что отображение $f_{l}$ можно понимать как преобразование, производимое $l$ тактами работы кодирующего устройства, полученного с помощью подключения входов булевой функции $f$ к некоторым ячейкам двоичного регистра сдвига.

Пусть $n \geqslant 1, p \geqslant 1, f \in \mathcal{F}_{n}, l \geqslant \max (1, p-n+2),\left(\tilde{x}_{1}, \tilde{x}_{2}, \ldots, \tilde{x}_{p}\right) \in V_{p}$. Через $f_{l, p}^{\left(\tilde{x}_{1}, \tilde{x}_{2}, \ldots, \tilde{x}_{p}\right)}$ будем обозначать отображение из $V_{l-p+n-1}$ в $V_{l}$, определяемое следующим образом:

$$
f_{l, p}^{\left(\tilde{x}_{1}, \tilde{x}_{2}, \ldots, \tilde{x}_{p}\right)}\left(x_{1}, x_{2}, \ldots, x_{l-p+n-1}\right) \equiv f_{l}\left(\tilde{x}_{1}, \tilde{x}_{2}, \ldots, \tilde{x}_{p}, x_{1}, x_{2}, \ldots, x_{l-p+n-1}\right) .
$$

Отображение $f_{l, p}^{\left(\tilde{x}_{1}, \tilde{x}_{2}, \ldots, \tilde{x}_{p}\right)}$ описывает преобразование, производимое $l$ тактами работы кодирующего устройства с функцией усложнения $f$ на двоичных последовательностях с фиксированным началом из $p$ символов. Особый интерес представляет случай $p=n-1$, при котором отображение данного типа действует из $V_{l}$ в $V_{l}$.

Определение 1 ([11]). Булева функция $f \in \mathcal{F}_{n}$ называется функиией дефекта нуль (функиией без запрета), если соотношение

$$
\left|f_{l}^{-1}(\mathbf{y})\right|>0
$$

выполняется для любого $l \geqslant 1$ и любого $\mathbf{y} \in V_{l}$.

Определение 2 ([11]). Булева функция $f \in \mathcal{F}_{n}$ называется совершенно уравновешенной, если соотношение

$$
\left|f_{l}^{-1}(\mathbf{y})\right|=2^{n-1}
$$

выполняется для любого $l \geqslant 1$ и любого $\mathbf{y} \in V_{l}$. Множество совершенно уравновешенных функций из $\mathcal{F}_{n}$ обозначается через $\mathcal{P} \mathcal{B}_{n}$.

Кодирующие устройства с совершенно уравновешенными функциями усложнения обладают следующим важным свойством: если на вход такого кодирующего устройства поступает чисто случайная последовательность, то выходная последовательность будет также чисто случайной.

Введем понятие барьера булевой функции, тесно связанное с понятием совершенной уравновешенности.

Определение 3 ([7]). Булева функция $f \in \mathcal{F}_{n}$ называется функцией с правым барьером длины $b$, если система уравнений

$$
\left\{\begin{array}{l}
f\left(x_{1}, x_{2}, \ldots, x_{n}\right)=f\left(z_{1}, z_{2}, \ldots, z_{n}\right) \\
f\left(x_{2}, x_{3}, \ldots, x_{n+1}\right)=f\left(z_{2}, z_{3}, \ldots, z_{n+1}\right) \\
\ldots \\
f\left(x_{b-1}, x_{b}, \ldots, x_{b+n-2}\right)=f\left(z_{b-1}, z_{b}, \ldots, z_{b+n-2}\right) \\
x_{1}=z_{1}, \ldots, x_{n-1}=z_{n-1}, x_{n}=0, z_{n}=1
\end{array}\right.
$$


имеет решение, а система

$$
\left\{\begin{array}{l}
f\left(x_{1}, x_{2}, \ldots, x_{n}\right)=f\left(z_{1}, z_{2}, \ldots, z_{n}\right) \\
f\left(x_{2}, x_{3}, \ldots, x_{n+1}\right)=f\left(z_{2}, z_{3}, \ldots, z_{n+1}\right) \\
\ldots \\
f\left(x_{b-1}, x_{b}, \ldots, x_{b+n-2}\right)=f\left(z_{b-1}, z_{b}, \ldots, z_{b+n-2}\right) \\
f\left(x_{b}, x_{b+1}, \ldots, x_{b+n-1}\right)=f\left(z_{b}, z_{b+1}, \ldots, z_{b+n-1}\right) \\
x_{1}=z_{1}, \ldots, x_{n-1}=z_{n-1}, x_{n}=0, z_{n}=1
\end{array}\right.
$$

решений не имеет.

Булева функция $f \in \mathcal{F}_{n}$ называется функцией с левым барьером длины $b$, если $\overleftrightarrow{f}\left(x_{1}, x_{2}, \ldots, x_{n}\right) \equiv f\left(x_{n}, x_{n-1}, \ldots, x_{1}\right)$ является функцией с правым барьером длины $b$.

Булева функция $f \in \mathcal{F}_{n}$ имеет барьер, если она имеет правый или левый барьер, или оба сразу. При этом длиной барьера функции называется соответственно длина правого барьера, левого барьера, или меньшая из длин барьеров.

Нам понадобятся также определения корреляционной иммунности и устойчивости булевых функций.

Определение 4 ([6]). Булева функция $f \quad \in \quad \mathcal{F}_{n}$ называется корреляционно-иммунной порядка $m, 0<m \leqslant n$, если для всяких $1 \leqslant i_{1}<i_{2}<\ldots<i_{m} \leqslant n$ и $\left(a_{1}, a_{2}, \ldots, a_{m}\right) \in V_{m}$ для функции $\tilde{f} \in \mathcal{F}_{n-m}$, полученной из $f$ фиксацией переменных с номерами $i_{1}, i_{2}, \ldots, i_{m}$ константами $a_{1}, a_{2}, \ldots, a_{m}$, выполнено соотношение

$$
\mathrm{wt}(\widetilde{f})=\frac{\mathrm{wt}(f)}{2^{m}} .
$$

Определение 5 ([6]). Булева функция $f \in \mathcal{F}_{n}$ для $0<m \leqslant n$ называется $m$-устойчивой, если она является уравновешенной и корреляционно-иммунной порядка $m$.

Пусть $n \in \mathbb{N}, f \in \mathcal{F}_{n}$. Обозначим для всякого $1 \leqslant i \leqslant n$ через $f^{(i)} \in \mathcal{F}_{n}$ функцию $f^{(i)}\left(x_{1}, x_{2}, \ldots, x_{i-1}, x_{i+1}, \ldots, x_{n}\right) \equiv f\left(x_{1}, x_{2}, \ldots, x_{i-1}, 0, x_{i+1}, \ldots, x_{n}\right)$.

Нетрудно видеть, что 1-устойчивость уравновешенной булевой функции $f$ от $n$ переменных эквивалентна тому, что для всякого $i, 1 \leqslant i \leqslant n$, функция $f^{(i)}$ является уравновешенной.

\section{3. Предварительные результаты}

Теорема 1 ([11]). Булева функиия совершенно уравновешена тогда и только тогда, когда она является функиией дефекта нуль (не имеет запрета).

Связь совершенной уравновешенности с наличием у функции барьера описывается следующим утверждением.

Теорема 2 ([7]). Если функция имеет барвер, то она совершенно уравновешена. 
Для всех утверждений, относящихся к функциям с правым барьером, очевидным образом могут быть построены аналоги для функций с левым барьером. Ввиду этого далее будем говорить только о правых барьерах функций, обозначая для удобства длину правого барьера булевой функции $f$ через $b_{f}^{R}$.

Для построения классов совершенно уравновешенных булевых функций удобно пользоваться следующей конструкцией. Пусть $g \in \mathcal{F}_{m}, h \in \mathcal{F}_{n}$. Тогда определим функцию $f=g[h] \in \mathcal{F}_{m+n-1}$ следующим образом:

$$
\begin{aligned}
f\left(x_{1}, \ldots, x_{m+n-1}\right)=g[ & h]\left(x_{1}, \ldots, x_{m+n-1}\right)= \\
& =g\left(h\left(x_{1}, \ldots, x_{n}\right), h\left(x_{2}, \ldots, x_{n+1}\right), \ldots, h\left(x_{m}, \ldots, x_{m+n-1}\right)\right) .
\end{aligned}
$$

Для данной конструкции верны следующие два утверждения.

Теорема 3 ([5]). Пусть $g \in \mathcal{F}_{m}, h \in \mathcal{F}_{n}$. Функиия $f=g[h] \in \mathcal{F}_{m+n-1}$ совершенно уравновешена тогда и только тогда, когда функиии $g$ и $h$ совершенно уравновешеHol.

Теорема 4 ([8]). Пусть $g \in \mathcal{F}_{m}, h \in \mathcal{F}_{n}, f=g[h]$. Тогда выполнено соотношение $\max \left\{b_{h}^{R}, b_{g}^{R}\right\} \leqslant b_{f}^{R} \leqslant b_{h}^{R}+b_{g}^{R}-1$.

В работе [9] получен ряд результатов о свойствах данной конструкции. Непосредственным образом из них вытекает следующее утверждение.

Теорема 5 ([9]). Пусть $m, n \in \mathbb{N}, g \in \mathcal{P B}_{m}, h^{(1)}, h^{(2)} \in \mathcal{F}_{n}, h^{(1)}(0,0, \ldots, 0)=$ $h^{(2)}(0,0, \ldots, 0)$. Ecли $h^{(1)} \neq h^{(2)}, \operatorname{mog} g\left[h^{(1)}\right] \neq g\left[h^{(2)}\right]$.

В работе [10] доказан ряд теорем о функциях с барьером, указывающих на нетривиальные структурные свойства порождаемых ими отображений.

Теорема 6 ([10]). Для каждой функиии $f \in \mathcal{F}_{n}$ с правым барьером можно определить такую величину $e_{f}^{R} \in\left\{0,1,2, \ldots, b_{f}^{R}-1\right\}$, что для любых $p \geqslant n-1, l \geqslant b_{f}^{R}+p-n$, $\left(\tilde{x}_{1}, \tilde{x}_{2}, \ldots, \tilde{x}_{p}\right) \in V_{p}$ и любого набора $\left(y_{1}, y_{2}, \ldots, y_{l}\right) \in \operatorname{Im}\left(f_{l, p}^{\left(\tilde{x}_{1}, \tilde{x}_{2}, \ldots, \tilde{x}_{p}\right)}\right)$ выполняется равенство

$$
\left|f_{l, p}^{\left(\tilde{x}_{1}, \tilde{x}_{2}, \ldots, \tilde{x}_{p}\right)^{-1}}\left(y_{1}, y_{2}, \ldots, y_{l}\right)\right|=2^{e_{f}^{R}} .
$$

Следствие 1 ([10]). Пусть $n \in \mathbb{N}, f \in \mathcal{F}_{n}$ имеет правый баръер. Для любых $l \geqslant b_{f}^{R}-1$ и любого набора $\left(\tilde{x}_{1}, \tilde{x}_{2}, \ldots, \tilde{x}_{n-1}\right) \in V_{n-1}$ верно равенство

$$
\left|\operatorname{Im}\left(f_{l, n-1}^{\left(\tilde{x}_{1}, \tilde{x}_{2}, \ldots, \tilde{x}_{n-1}\right)}\right)\right|=2^{l-e_{f}^{R}} .
$$

\section{4. Основные результаты}

Отсутствие проработанного аппарата изучения совершенно уравновешенных булевых функций часто приводило к попыткам сводить их исследование к другим важным для криптографических приложений свойствам, более сильным, чем уравновешенность. В работах $[1,2,3]$ большое внимание уделяется подфункциям совершенно уравновешенных функций. 
Тем не менее, данный путь изучения совершенно уравновешенных функций является чересчур узким. Ярким примером этого является полученное в работе [2] Маркусом Дихтлом утверждение об уравновешенности подфункций совершенно уравновешенных булевых функций, содержащее существенный пробел в доказательстве.

Теорема (опровергнутая) [2]. Пусть $n \in \mathbb{N}, f \in \mathcal{F}_{n}$. Если $f$ является совершенно уравновешенной, то существует не более одного такого $j(1 \leqslant j \leqslant n)$, что функиия $f^{(j)} \in \mathcal{F}_{n-1}, f^{(j)}\left(x_{1}, \ldots, x_{j-1}, x_{j+1}, \ldots, x_{n}\right) \equiv f\left(x_{1}, \ldots, x_{j-1}, 0, x_{j+1}, \ldots, x_{n}\right)$ (подфункиия $f$, полученная фиксацией $j$-й переменной константой 0 ), не является уравновешенной.

В работе [4] был построен частный пример, опровергающий утверждение Маркуса Дихтла (совершенно уравновешенная функция от 5 переменных, две подфункции которой, полученные фиксацией одной из двух разных переменных нулями, не являются уравновешенными). Теоремы 3 и 5 позволяют построить последовательность широких классов совершенно уравновешенных функций, противоречащих утверждению Дихтла.

Теорема 7. При всяком $n \geqslant 7$ существует не менее $2^{2^{n-6}}-1$ булевых функций из $\mathcal{P B}_{n}$, противоречащих утверждению Дихтла.

Доказательство. Рассмотрим для произвольного $n \geqslant 7$ следующее множество функций:

$$
\begin{array}{r}
\left\{f=g[h] \mid g\left(z_{1}, z_{2}, z_{3}\right)=z_{1} \oplus z_{2} z_{3}, h\left(x_{1}, x_{2}, \ldots, x_{n-2}\right)=\right. \\
\left.=r\left(x_{1}, x_{2}, \ldots, x_{n-6}\right) \cdot x_{n-4} x_{n-3} \oplus x_{n-2}\right\},
\end{array}
$$

где $r\left(x_{1}, x_{2}, \ldots, x_{n-6}\right)$ - произвольная функция, отличная от нулевой. Легко видеть, что функция $g$ имеет левый барьер длины 1 , а каждая из функций $h$ имеет правый барьер длины 1 , то есть все они являются совершенно уравновешенными.

Из Теоремы 5 следует, что все функции в данном множестве различны и являются совершенно уравновешенными. Покажем теперь, что для каждой из них не выполнено условие Дихтла.

Для этого достаточно показать, что для произвольной функции $f=g[h]$, $g\left(z_{1}, z_{2}, z_{3}\right)=z_{1} \oplus z_{2} z_{3}, h\left(x_{1}, x_{2}, \ldots, x_{n-2}\right)=r\left(x_{1}, x_{2}, \ldots, x_{n-6}\right) \cdot x_{n-4} x_{n-3} \oplus x_{n-2}$, подфункции $f^{(n-2)}$ и $f^{(n)}$ не являются уравновешенными. Покажем это.

1) $f^{(n-2)}\left(x_{1}, x_{2}, \ldots, x_{n-3}, x_{n-1}, x_{n}\right)=r\left(x_{1}, x_{2}, \ldots, x_{n-6}\right) \cdot x_{n-4} x_{n-3} \oplus x_{n-1} x_{n}$ и

$$
\begin{aligned}
& \operatorname{wt}\left(f^{(n-2)}\right)=\operatorname{wt}\left(r\left(x_{1}, x_{2}, \ldots, x_{n-6}\right) \cdot x_{n-4} x_{n-3}\right)+\operatorname{wt}\left(x_{n-1} x_{n}\right)- \\
& -2 \operatorname{wt}\left(r\left(x_{1}, x_{2}, \ldots, x_{n-6}\right) \cdot x_{n-4} x_{n-3} x_{n-1} x_{n}\right)=8 \operatorname{wt}(r)+2^{n-3}-4 \operatorname{wt}(r)= \\
& =2^{n-3}+4 \operatorname{wt}(r) \leqslant 2^{n-3}+4 \cdot 2^{n-6}<2^{n-2},
\end{aligned}
$$

следовательно, $f^{(n-2)}$ не уравновешена.

2) $f^{(n)}\left(x_{1}, x_{2}, \ldots, x_{n-1}\right)=r\left(x_{1}, x_{2}, \ldots, x_{n-6}\right) \cdot x_{n-4} x_{n-3} \oplus x_{n-2} \oplus r\left(x_{2}, x_{3}, \ldots, x_{n-5}\right)$. $x_{n-3} x_{n-2} \cdot r\left(x_{3}, x_{4}, \ldots, x_{n-4}\right) \cdot x_{n-2} x_{n-1} \oplus x_{n-2} \cdot r\left(x_{3}, x_{4}, \ldots, x_{n-4}\right) \cdot x_{n-2} x_{n-1}$ 
и

$$
\begin{aligned}
& \operatorname{wt}\left(f^{(n)}\right)=\operatorname{wt}\left(f^{(n)}\left(x_{1}, x_{2}, \ldots, x_{n-3}, 0, x_{n-1}\right)\right)+\operatorname{wt}\left(f^{(n)}\left(x_{1}, x_{2}, \ldots, x_{n-3}, 1,0\right)\right)+ \\
& +\operatorname{wt}\left(f^{(n)}\left(x_{1}, x_{2}, \ldots, 0,1,1\right)\right)+\operatorname{wt}\left(f^{(n)}\left(x_{1}, x_{2}, \ldots, 1,1,1\right)\right)= \\
& =\operatorname{wt}\left(r\left(x_{1}, x_{2}, \ldots, x_{n-6}\right) \cdot x_{n-4} x_{n-3}\right)+ \\
& +\operatorname{wt}\left(r\left(x_{1}, x_{2}, \ldots, x_{n-6}\right) \cdot x_{n-4} x_{n-3} \oplus 1\right)+\operatorname{wt}\left(r\left(x_{3}, x_{2}, \ldots, x_{n-4}\right) \oplus 1\right)+ \\
& +\operatorname{wt}\left(r\left(x_{1}, x_{2}, \ldots, x_{n-6}\right) \cdot x_{n-4} \oplus 1 \oplus r\left(x_{2}, \ldots, x_{n-5}\right) \cdot r\left(x_{3}, x_{2}, \ldots, x_{n-4}\right)\right)= \\
& =4 \operatorname{wt}(r)+2^{n-3}-2 \operatorname{wt}(r)+2^{n-4}-4 \operatorname{wt}(r)+ \\
& +2^{n-4}-\operatorname{wt}\left(r\left(x_{1}, \ldots, x_{n-6}\right) \cdot x_{n-4} \oplus r\left(x_{2}, \ldots, x_{n-5}\right) \cdot r\left(x_{3}, x_{2}, \ldots, x_{n-4}\right)\right) \leqslant \\
& \leqslant 2^{n-2}-2 \operatorname{wt}(r)<2^{n-2},
\end{aligned}
$$

следовательно, $f^{(n)}$ также не уравновешена.

Таким образом, для всякого $n \geqslant 7$ построен класс из $2^{2^{n-6}}-1$ функций, каждая из которых является совершенно уравновешенной, но содержит не менее двух аргументов, фиксация которых приводит к функции, не являющейся уравновешенной (корреляционная иммунность означает отсутствие таких переменных).

Обратимся теперь к такому классу совершенно уравновешенных функций, как функции с барьером. Опишем их структурные свойства, которые помогут нам доказать положительное утверждение о корреляционной иммунности функций.

Лемма 1. Пусть $f \in \mathcal{F}_{n}$ и $b_{f}^{R}<+\infty$. Для всякого набора $\left(a_{1}, a_{2}, \ldots, a_{n-1}\right) \in V_{n-1}$, любого натурального $k$ и всякого $\left(z_{1}, z_{2}, \ldots, z_{k}\right) \in V_{k}$ верно:

$\left(y_{1}, y_{2}, \ldots, y_{b_{f}^{R}-1}\right)$ лежит в образе отображения $f_{b_{f}^{R}-1, n-1}^{\left(a_{1}, a_{2}, \ldots, a_{n-1}\right)}$ тогда и только тогда, когда $\left(y_{1}, y_{2}, \ldots, y_{b_{f}^{R}-1}, z_{1}, z_{2}, \ldots, z_{k}\right)$ лежит в образе отображения $f_{b_{f}^{R}-1+k, n-1}^{\left(a_{1}, a_{2}, \ldots, a_{n-1}\right)}$.

Доказательство. Для произвольного набора $\left(y_{1}, y_{2}, \ldots, y_{b_{f}^{R}-1}, z_{1}, z_{2}, \ldots, z_{k}\right)$ из принадлежности множеству $\operatorname{Im}\left(f_{b_{f}^{R}-1+k, n-1}^{\left(a_{1}, a_{2}, \ldots, a_{n-1}\right)}\right)$ непосредственно следует принадлежность его начального отрезка $\left(y_{1}, y_{2}, \ldots, y_{b_{f}^{R}-1}\right)$ множеству $\operatorname{Im}\left(f_{b_{f}^{R}-1, n-1}^{\left(a_{1}, a_{2}, \ldots, a_{n-1}\right)}\right)$.

Обратно. Если

$$
\left|f_{b_{f}^{R}-1, n-1}^{\left(a_{1}, a_{2}, \ldots, a_{n-1}\right)^{-1}}\left(y_{1}, y_{2}, \ldots, y_{b_{f}^{R}-1}\right)\right|>0
$$

то, с учетом наличия у $f$ правого барьера длины $b_{f}^{R}$ и Теоремы 6 ,

$$
\left|f_{b_{f}^{R}-1, n-1}^{\left(a_{1}, a_{2}, \ldots, a_{n-1}\right)^{-1}}\left(y_{1}, y_{2}, \ldots, y_{b_{f}^{R}-1}\right)\right|=2^{e_{f}^{R}}
$$

откуда

$$
\sum_{\left(z_{1}, z_{2}, \ldots, z_{k}\right) \in V_{k}}\left|f_{b_{f}^{R}-1+k, n-1}^{\left(a_{1}, a_{2}, \ldots, a_{n-1}\right)^{-1}}\left(y_{1}, y_{2}, \ldots, y_{b_{f}^{R}-1}, z_{1}, z_{2}, \ldots, z_{k}\right)\right|=2^{e_{f}^{R}} \cdot 2^{k} .
$$

Так как по Теореме 6 для всякого $\left(z_{1}, z_{2}, \ldots, z_{k}\right) \in V_{k}$ мощность

$$
f_{b_{f}^{R}-1+k, n-1}^{\left(a_{1}, a_{2}, \ldots, a_{n-1}\right)^{-1}}\left(y_{1}, y_{2}, \ldots, y_{b_{f}^{R}-1}, z_{1}, z_{2}, \ldots, z_{k}\right)
$$


может быть равна только 0 или $2^{e_{f}^{R}}$, получим, что для всякого $\left(z_{1}, z_{2}, \ldots, z_{k}\right) \in V_{k}$ она равна в точности $2_{f}^{e_{f}^{R}}$ и $\left(y_{1}, y_{2}, \ldots, y_{b_{f}^{R}-1}, z_{1}, z_{2}, \ldots, z_{k}\right)$ лежит в образе отображения $f_{b_{f}^{R}-1+k, n-1}^{\left(a_{1}, a_{2}, \ldots, a_{n-1}\right)}$

Теорема 8. Пусть $f \in \mathcal{F}_{n} u b_{f}^{R} \leqslant n-1$. Для всякого натурального $r \leqslant n-b_{f}^{R}$, любого набора $\left(a_{1}, a_{2}, \ldots, a_{r}\right) \in V_{r}$ и всякого натурального $k$ отображение $f_{k, r}^{\left(a_{1}, a_{2}, \ldots, a_{r}\right)}$ : $V_{k+(n-1-r)} \rightarrow V_{k}$ является уравновешенным.

Доказательство. Для любых $k \in \mathbb{N}$ и $\left(z_{1}, z_{2}, \ldots, z_{k}\right) \in V_{k}$ рассмотрим прообраз $\left(z_{1}, z_{2}, \ldots, z_{k}\right)$ относительно отображения $f_{k, r}^{\left(a_{1}, a_{2}, \ldots, a_{r}\right)}$. Зафиксируем произвольным образом набор $\left(b_{1}, b_{2}, \ldots, b_{n-1-r}\right) \in V_{n-1-r}$ и получим следующую цепочку равенств:

$$
\begin{aligned}
& \left|f_{k, r}^{\left(a_{1}, a_{2}, \ldots, a_{r}\right)^{-1}}\left(z_{1}, z_{2}, \ldots, z_{k}\right)\right|=\mid\left\{\left(x_{1}, x_{2}, \ldots, x_{k+(n-1-r)}\right) \in V_{k+(n-1-r)}:\right. \\
& \left.f_{k}\left(a_{1}, a_{2}, \ldots, a_{r}, x_{1}, x_{2}, \ldots, x_{k+(n-1-r)}\right)=\left(z_{1}, z_{2}, \ldots, z_{k}\right)\right\} \mid= \\
& \sum_{\left(y_{1}, y_{2}, \ldots, y_{n-1-r}\right) \in V_{n-1-r}} \mid\left\{\left(x_{1}, x_{2}, \ldots, x_{k+(n-1-r)}\right) \in V_{k+(n-1-r)}:\right. \\
& f_{k+(n-1-r)}\left(b_{1}, b_{2}, \ldots, b_{n-1-r}, a_{1}, a_{2}, \ldots, a_{r}, x_{1}, x_{2}, \ldots, x_{k+(n-1-r)}\right)= \\
& \left.=\left(y_{1}, y_{2}, \ldots, y_{n-1-r}, z_{1}, z_{2}, \ldots, z_{k}\right)\right\} \mid=
\end{aligned}
$$

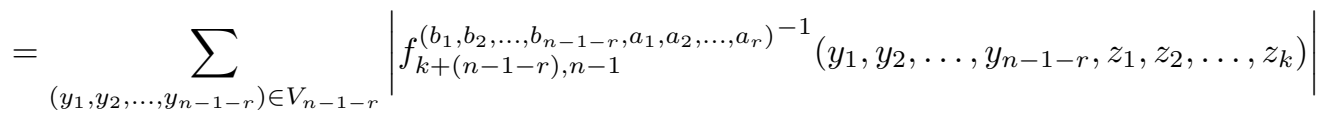

Пользуясь Леммой 1 с учетом неравенства $n-1-r \geqslant b_{f}^{R}-1$ получим, что последнее выражение равно

$$
\sum_{\substack{\left(y_{1}, y_{2}, \ldots, y_{n-1-r}\right) \in \\
\operatorname{Im}\left(b_{1}^{\left(b_{1}, b_{2}, \ldots, b_{n-1-r}, a_{1}, a_{2}, \ldots, a_{r}\right)}\right)}} \mid \begin{aligned}
& f_{k+(n-1-r), n-1}^{\left(b_{1}, b_{2}, \ldots, b_{n-1-r}, a_{1}, a_{2}, \ldots, a_{r}\right)^{-1}}\left(y_{1}, y_{2}, \ldots, y_{n-1-r}, z_{1}, z_{2}, \ldots, z_{k}\right) \mid . \\
& f_{-1-r, n-1}
\end{aligned}
$$

Учитывая, что $k+n-1-r \geqslant b_{f}^{R}-1$, воспользуемся также Теоремой 6 и получим, что последнее выражение равно $\left|\operatorname{Im}\left(f_{n-1-r, n-1}^{\left(b_{1}, b_{2}, \ldots, b_{n-1-r}, a_{1}, a_{2}, \ldots, a_{r}\right)}\right)\right| \cdot 2^{e_{f}^{R}}$, что, с учетом неравенства $n-1-r \geqslant b_{f}^{R}-1$ и Следствия 1 , равно $2^{n-1-r-e_{f}^{R}} \cdot 2^{e_{f}^{R}}=2^{n-1-r}$.

Полагая $k=1$ в Теореме 8 , получим следующее утверждение об уравновешенности подфункций функций с барьером.

Следствие 2. Пусть $f \in \mathcal{F}_{n} u b_{f}^{R} \leqslant n-1$. Для всякого натурального $r \leqslant n-b_{f}^{R} u$ любого набора $\left(a_{1}, a_{2}, \ldots, a_{r}\right) \in V_{r}$ подфункиия

$$
\widetilde{f}\left(x_{1}, x_{2}, \ldots, x_{n-r}\right) \equiv f\left(a_{1}, a_{2}, \ldots, a_{r}, x_{1}, x_{2}, \ldots, x_{n-r}\right)
$$

является уравновешенной.

Используя полученные результаты, окончательно докажем утверждение, связывающее свойства совершенно уравновешенных булевых функций и свойство корреляционной иммунности (устойчивости). 
Теорема 9. Пусть $f \in \mathcal{F}_{n} u b_{f}^{R}+b_{f}^{L} \leqslant n$. Тогда $f$ является 1-устойчивой функицей.

Доказательство. Рассмотрим некоторую функцию $f \in \mathcal{F}_{n}$, для которой выполняется условие $b_{f}^{R}+b_{f}^{L} \leqslant n$. Так как функция $f$ имеет барьер, то она является совершенно уравновешенной и, следовательно, ее вес равен $2^{n-1}$. Таким образом, доказываемое утверждение эквивалентно тому, что для любого $i, i=1,2, \ldots, n$, функция $f^{(i)}$ имеет вес $2^{n-2}$. Покажем это для произвольного $i, i=1,2, \ldots, n$.

Рассмотрим вначале случай $i \leqslant n-b_{f}^{R}$. Тогда по Следствию 2 для всякого набора $\left(a_{1}, a_{2}, \ldots, a_{i}\right) \in V_{i}$ верно $\left|\left(f_{1, i}^{\left(a_{1}, a_{2}, \ldots, a_{i}\right)}\right)^{-1}(1)\right|=2^{n-i-1}$. Тогда, очевидно,

$$
\left(f^{(i)}\right)^{-1}(1)=\bigcup_{\left(a_{1}, a_{2}, \ldots, a_{i-1}\right) \in V_{i-1}}\left\{\left(c_{1}, c_{2}, \ldots, c_{n}\right) \in V_{n}:\left(c_{1}, c_{2}, \ldots, c_{i}\right)=\left(a_{1}, a_{2}, \ldots, a_{i-1}, 0\right),\right.
$$

$$
\left.\left(c_{i+1}, c_{i+2}, \ldots, c_{n}\right) \in\left(f_{1, i}^{\left(a_{1}, a_{2}, \ldots, a_{i}\right)}\right)^{-1}(1)\right\}
$$

и, следовательно, $\operatorname{wt}\left(f^{(i)}\right)=\left|\left(f^{(i)}\right)^{-1}(1)\right| \underset{\left(a_{1}, a_{2}, \ldots, a_{i-1}\right) \in V_{i-1}}{\sum_{n-i-1}}=2^{i-1} \cdot 2^{n-i-1}=$ $2^{n-2}$.

Пусть теперь $i \geqslant n-b_{f}^{R}+1$. Тогда по условию верно $i \geqslant b_{f}^{L}+1$. Рассмотрим функцию $\overleftrightarrow{f}\left(x_{1}, x_{2}, \ldots, x_{n}\right)=f\left(x_{n}, x_{n-1}, \ldots, x_{1}\right)$, для которой, как следует из определения правого барьера, $b \underset{f}{R}=b_{f}^{L}$. Очевидно, что $f^{(i)}\left(x_{1}, x_{2}, \ldots, x_{i-1}, x_{i+1}, \ldots, x_{n}\right)=$ $\overleftrightarrow{f}(n+1-i)\left(x_{n}, x_{n-1}, \ldots, x_{i+1}, x_{i-1}, \ldots, x_{1}\right)$. Так как $i \geqslant b_{f}^{L}+1$, то $n+1-i \leqslant$ $n+1-b_{f}^{L}-1=n-b_{f}^{L}=n-b \underset{f}{R}$; отсюда и из приведенных выше рассмотрений следует равенство $\operatorname{wt}(\overleftrightarrow{f}(n+1-i))=2^{n-2}$, что и означает $\operatorname{wt}\left(f^{(i)}\right)=2^{n-2}$.

\section{Список литературы}

1. Anderson R.J., "Searching for the optimum correlation attack", FSE 1995, Lect. Notes Comput. Sci., 1008, 1995, 137-143.

2. Dichtl M., "On nonlinear filter generators", FSE 1997, Lect. Notes Comput. Sci., 1267, Springer, Heidelberg, 1997, 103-106.

3. Gouget A., Sibert H., "Revisiting correlation-immunity in filter generators", SAC 2007, Lect. Notes Comput. Sci., 4876, 2007, 378-395.

4. Smyshlyaev S. V., "Perfectly balanced Boolean functions and Golić conjecture", J. Cryptology, 25:3 (2012), 464-483.

5. Логачев О. А., "Об одном классе совершенно уравновешенных булевых функций”, Матер. 3-й междунар. научн. конф. по пробл. безопасн. противод. террор. (МЦНМО), $2008,137-141$.

6. Логачев О.А., Сальников А.А., Смышляев С.В., Ященко В.В., Булевы функиии в теории кодирования и криптологии, 3-е издание, М.: ЛЕНАНД, 2015.

7. Логачев О.А., Смышляев С. В., Ященко В.В., "Новые методы изучения совершенно уравновешенных булевых функций", Дискретная математика, 21:2 (2009), 51-74; англ. пер.: Logachev O. A., Smyshlyaev S. V., Yashchenko V. V., "New methods of investigation of perfectly balanced Boolean functions", Discrete Math. Appl., 19:3 (2009), $237-262$. 
8. Смышляев С. В., “Барьеры совершенно уравновешенных булевых функций”, Дискретная математика, 22:2 (2010), 66-79; англ. пер.: Smyshlyaev S. V., "Barriers of perfectly balanced Boolean functions", Discrete Math. Appl., 20:3 (2010), 321-336.

9. Смышляев С. В., "Построение классов совершенно уравновешенных булевых функций без барьера", Прикладная дискретная математика, 2010, №3(9), 41-50.

10. Смышляев С. В., "Булевы функции без предсказывания", Дискретная математика, 23:1 (2011), 102-118; англ. пер.: Smyshlyaev S. V., "Boolean functions without prediction", Discrete Math. Appl., 21:2 (2011), 209-227.

11. Сумароков С.Н., “Запреты двоичных функций и обратимость для одного класса кодирующих устройств", Обоз. прикл. промышл. матем., 1:1 (1994), 33-55.

Статья поступила 19.04.2016. 